\title{
Electron Confinement in Surface States on a Stepped Gold Surface Revealed by Angle-Resolved Photoemission
}

\author{
A. Mugarza, ${ }^{1,2}$ A. Mascaraque, ${ }^{3}$ V. Pérez-Dieste, ${ }^{3}$ V. Repain, ${ }^{4}$ S. Rousset, ${ }^{4}$ F. J. García de Abajo, ${ }^{5}$ and J. E. Ortega ${ }^{1,2}$ \\ ${ }^{1}$ Departamento de Física Aplicada I, Universidad del País Vasco, Plaza Oñati 2, 20018 San Sebastian, Spain \\ ${ }^{2}$ Donostia International Physics Center (DIPC), Manuel Lardizabal 4, 20018 San Sebastian, Spain \\ ${ }^{3}$ LURE Centre Universitaire Paris-Sud, Bâtiment 209D B.P. 34, 91898 Orsay Cedex, France \\ ${ }^{4}$ Groupe de Physique des Solides, Universités Paris 6 et 7, 2 Place Jussieu, 75251 Paris Cedex 5, France \\ ${ }^{5}$ Centro Mixto CSIC-UPV/EHU, Manuel Lardizabal 3, 20018 San Sebastián, Spain
}

(Received 27 February 2001; published 17 August 2001)

STM images show that vicinal Au(788) surfaces are made up of a uniform array of (111)-oriented terraces of similar width $(\sim 3.8 \mathrm{~nm})$. This uniformity makes it possible to study the electronic structure of the resulting step superlattice by angle-resolved photoemission. We show that for this terrace array the surface state appears to be broken up into one-dimensional quantum-well levels, indicating total electron confinement within the terraces. The angular resolution allows the probability density of the terrace quantum well state to be mapped in reciprocal space, complementing nicely the wave function measured in real space by STM.

DOI: $10.1103 /$ PhysRevLett.87.107601

PACS numbers: 79.60.Bm, 68.35.Bs, 73.21.-b

Vicinal surfaces are natural templates for growing nanostructured solids with low-dimensional properties $[1,2]$. They also serve as model systems for studying the electron wave functions and other basic properties of lateral nanostructures [3]. Vicinal noble metal (111) surfaces are particularly suitable because they have a freeelectron-like surface state that scatters strongly at step edges, leading to one-dimensional confinement within terraces, standing-wave patterns, and step superlattice effects [4-6]. One big advantage is that the surface state can be readily probed by both scanning tunneling microscopy/ spectroscopy (STM/STS) or photoemission [3-7]. Since photoemission is an averaging technique it requires regular arrays of steps over comparatively large areas to obtain meaningful results. However, most surfaces have a variety of defects and in general a broad distribution of terrace sizes. This hampers the observation of small effects such as the opening of a step superlattice gap at the edge of the Brillouin zone. In contrast, in STM a single terrace is enough to study electronic properties, such as confinement or scattering, but the reciprocal space of the superlattice cannot be properly investigated as in photoemission. It is interesting to note the difference between STM experiments that reveal total electron confinement $[4,5]$, and photoemission results that show electron dispersion across the steps and superlattice effects [3,6,7]. Although the differences appear to be related to the average terrace size involved (large in STM, small in photoemission, [3]), in fact terrace confinement of surface states observed with photoemission has never been reported previously. Here we present clear evidence of the first two energy levels of a terrace quantum well from photoemission in agreement with STM observations. Both levels display strong angular-dependent intensity, as expected from the photoemission matrix element of one-dimensional quantum-well levels. We show how angular scans of the photoemission intensity probe in reciprocal space the same localized electron wave functions probed by STM in real space.

Photoemission experiments have been performed at the SU8 undulator, Spanish-French beam line at LURE (Paris). The experiments were performed at $300 \mathrm{~K}$ using $p$-polarized light and photon energy $h \nu=27 \mathrm{eV}$. The experimental station is equipped with an angle-resolved photoemission setup that provides an angular resolution lower than $0.5^{\circ}$. The total energy resolution is $50 \mathrm{meV}$. The high index surface is $\mathrm{Au}(788)$, i.e., $\mathrm{Au}(111)$ vicinal with a miscut angle of $3.5^{\circ}$ towards [211] (composed of $\{111\}$-like steps). The single crystal was prepared by extensive sputtering-annealing cycles until the LEED pattern displayed the characteristic spot splitting. Figure 1a shows a STM picture of the $\mathrm{Au}(788)$ on a large scale. In the bottom right the inset shows a zoom of a few terraces where the corrugation due to the terrace levels has been subtracted. This enhances the discommensuration lines running perpendicular to the step edges that indicate the presence of alternating fcc- and hcp-packed domains along a single terrace. Figure $1 \mathrm{~b}$ shows the terrace width distribution of the surface measured over 30 images and more than 200 terraces. $60 \%$ of the terraces have the nominal $L=38.2 \AA$ terrace width \pm one atomic row. The reason for such a homogeneous distribution at room temperature must be related to the high kink energy of the $\{111\}$ steps on gold. One may invoke also the presence of the surface reconstruction stabilizing straight steps as observed on $\mathrm{Si}(111)$ [8]. For most of the $\mathrm{Au}(111)$ vicinals this reconstruction leads to a hill-and-valley, faceted structure [9], but $\mathrm{Au}(788)$ is a stable orientation. The LEED pattern of the specular spot taken at $E=20 \mathrm{eV}$ shown in Fig. 1c demonstrates on a macroscopic scale the excellent periodicity of the surface in both the direction 
(a)

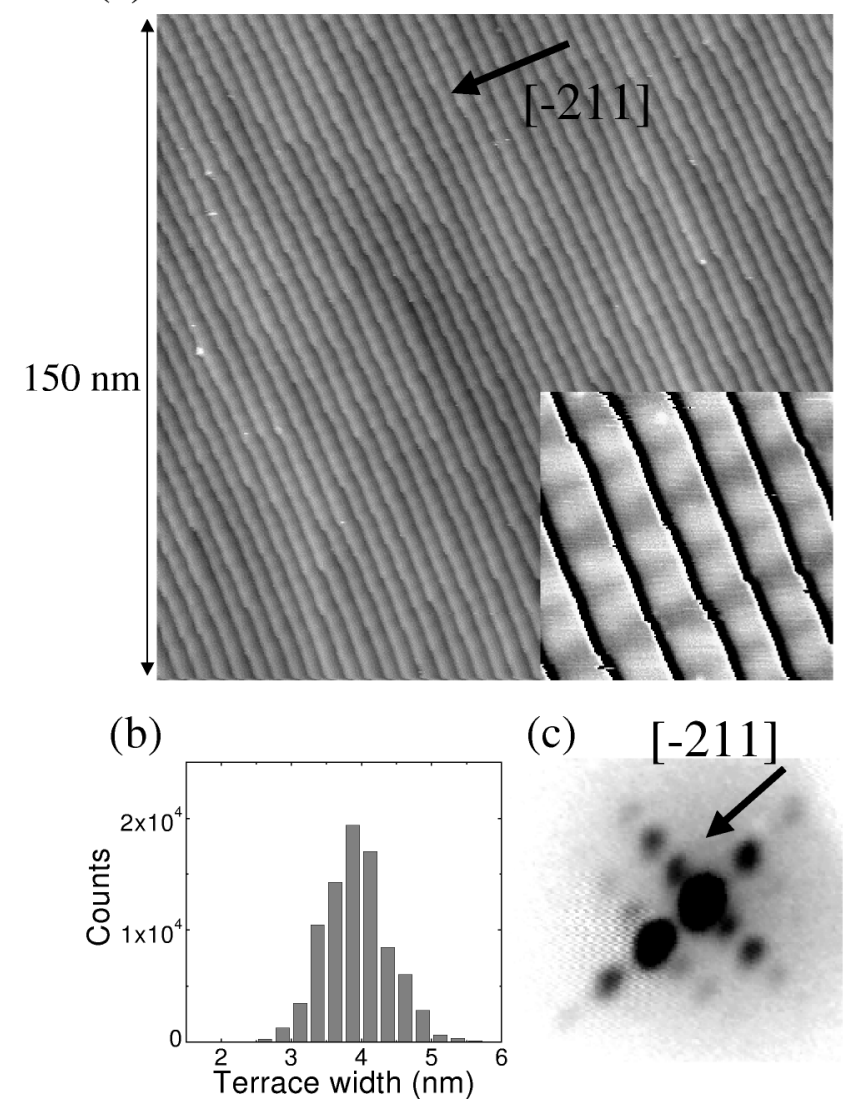

FIG. 1. (a) STM image of $\mathrm{Au}(788)$ showing straight monatomic steps and (b) the corresponding terrace width distribution. The inset shows the detailed structure at a smaller scale. Bright and dark areas indicate the presence of alternating fccand hcp-packed layers within the same terrace [9]. (c) The LEED pattern contains both superstructures perpendicular and parallel to the terraces.

perpendicular (narrow terrace width distribution) and parallel (72 $\AA$ periodic fcc-hcp reconstruction) to the steps.

Figure 2 shows the photoemission spectra of $\mathrm{Au}(788)$ near the Fermi level and the dispersion of the surface state peak along the two relevant directions of the surface, i.e., perpendicular ( $x$ direction) and parallel ( $y$ direction) to the steps. $k_{x}$ and $k_{y}$ are straightforwardly obtained from the emission angle and the (measured) kinetic energy $k_{x, y}=\sqrt{\left(2 m / \hbar^{2}\right) E_{\text {kin }}} \sin \theta$. The emission angle is measured with respect to the [788] surface normal direction. The anisotropy of the dispersion in Fig. 2 is striking. Along the steps the surface state behaves as in flat $\mathrm{Au}(111)$. It disperses symmetrically around $k_{y}=0$, and crosses the Fermi level at $k_{y}= \pm 0.168 \AA^{-1}$. The resulting effective mass $\left(m^{*}=0.27 \times m_{e}\right)$ is similar to $\mathrm{Au}(111)$ [10]. In the perpendicular direction the main surface peak does not show any appreciable dispersion over a very wide angular range. Therefore the surface state in Fig. 2 has onedimensional character, i.e., it has a free-electron-like dispersion along the terraces and it becomes totally confined

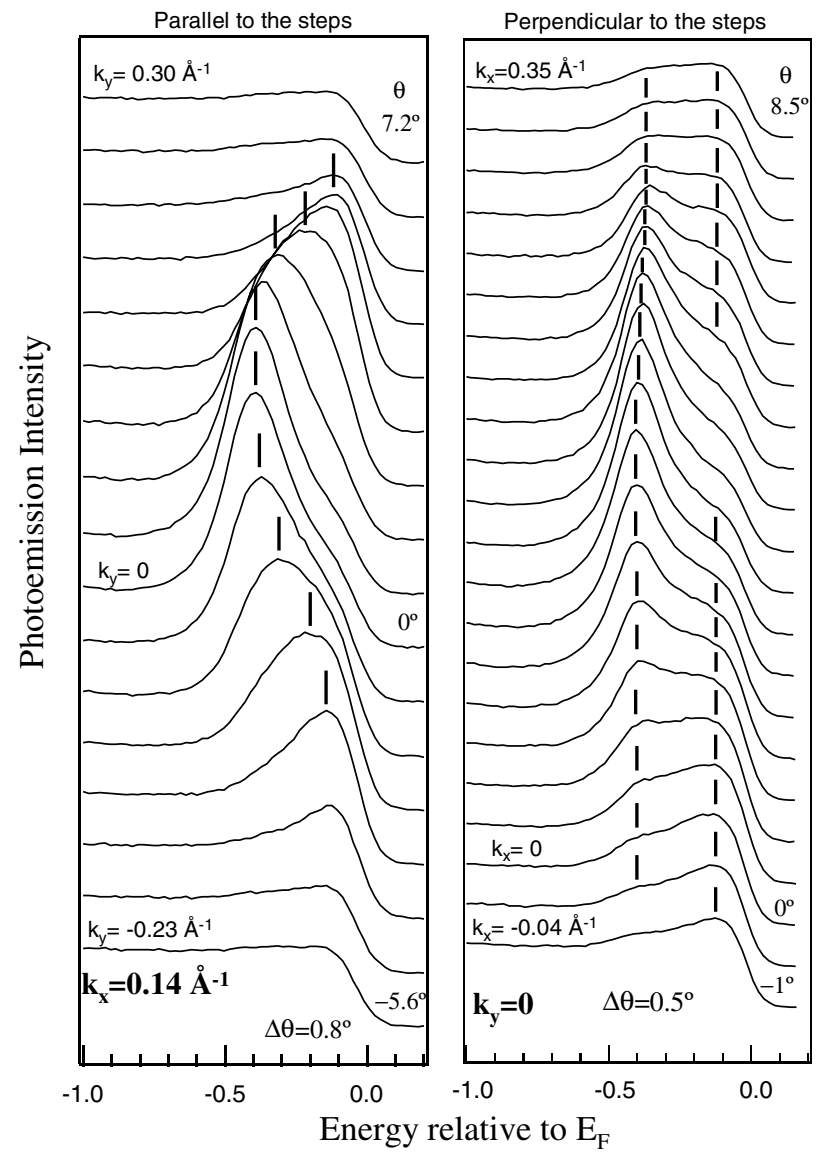

FIG. 2. Dispersion of the surface state at $\mathrm{Au}(788)$ parallel (left) and perpendicular (right) to the step superlattice. The strong asymmetry indicates the presence of a one-dimensional surface state, i.e., a free-electron-like state parallel to the steps, but confined in the perpendicular direction where it splits in two different quantum levels

between two steps in the perpendicular direction, as observed in STM $[4,5]$.

The dispersion across the steps in the right panel of Fig. 2 shows a second feature (tick marks) at about $-0.1 \mathrm{eV}$ which is more intense a few degrees off normal emission. In order to extract the details of this second peak we have performed a line fitting to the spectra. The fit was done using Lorentzians for both peaks plus a Shirley and a linear background, all convoluted with a Gaussian to account for the overall energy resolution. From this fit we obtained the binding energy of both peaks, shown in Fig. 3, and their intensity (area under the peak), shown in Fig. 4. The size of the data points in Fig. 3 reflects the intensity of the peaks shown in Fig. 4. Figure 3 also includes the parabolic dispersion in the direction parallel to the steps using the same wave vector scale for $k_{y}$. Within the error bars, the fit gives two flat bands at -0.40 and $-0.11 \mathrm{eV}$. The lack of dispersion proves the presence of quantum levels in a totally-confining potential well, as observed in STM experiments on $\operatorname{Ag}(111)$ [5]. Furthermore, the energy gap in Fig. 3 gives exactly the value of the energy interval between the lowest two levels of the infinite 


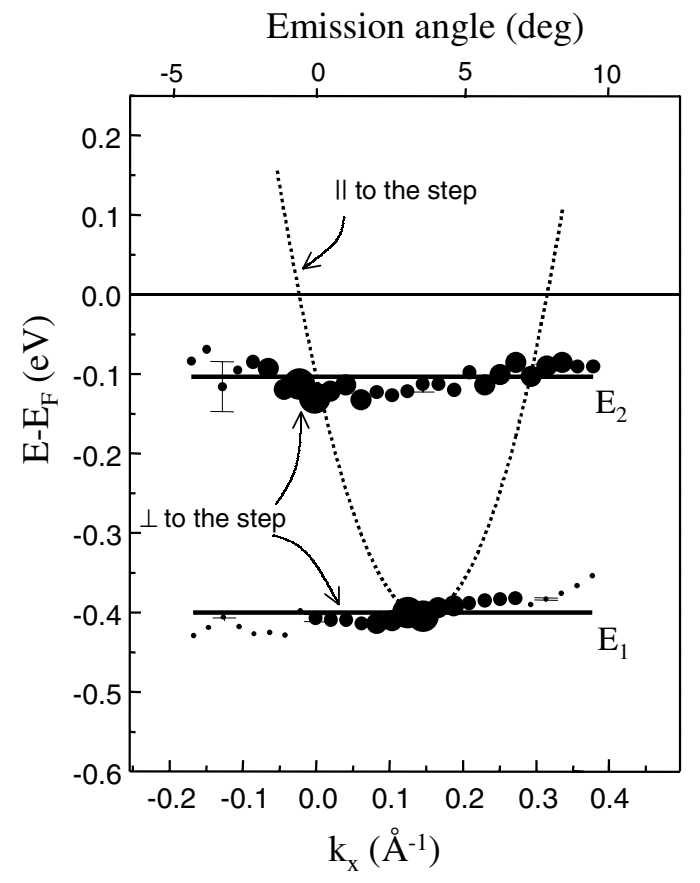

FIG. 3. Surface state energy as a function of the wave vector parallel to the (111) terrace, and perpendicular $\left(k_{y}=0\right.$, data points) and parallel ( $k_{x}=0.14 \AA^{-1}$, dotted line) to the steps, obtained from the data in Fig. 2. The size of the dots is proportional to their corresponding peak intensity (Fig. 4). The energy gap between quantum levels and the lack of dispersion indicates total electron confinement within terraces.

one-dimensional quantum well of size $L=38.2 \AA$, i.e., $\left(2^{2}-1^{2}\right) \times\left(\hbar^{2} \pi^{2}\right) /\left(2 m^{*} L^{2}\right)=0.29 \mathrm{eV}$, where $m^{*}=$ $0.27 \times m_{e}$. This gap is equal to the one measured by STM at the center of a slightly narrower terrace $(36 \AA)$ on $\mathrm{Au}(111)$, though the first two peaks in the differential conductance spectra of Ref. [4] lie at -0.33 and $-0.01, \sim 0.1 \mathrm{eV}$ above the quantum levels of Fig. 3. Full confinement indicates that there is no coupling between terraces for $\mathrm{Au}(788)$, i.e., the transmission of the surface wave function across the step potential barrier is small [11]. In contrast, transmission and coupling between terraces is needed to explain the band dispersion observed on vicinal $\mathrm{Cu}(111)$ surfaces with higher step densities [6,7]. Such differences must be associated with the switch from terrace to step modulation of the surface wave function observed on vicinal $\mathrm{Cu}(111)$ as the step density increases [3].

The energy of the $N$ th level of the terrace quantum well is referred to $E_{F}-E_{0}$, i.e., the ground state of the flat surface $(L=\infty)$ :

$$
E_{N}=E_{F}-E_{0}+\frac{\hbar^{2} \pi^{2}}{2 m^{*} L^{2}} N^{2} .
$$

From the absolute energy of the quantum levels in Fig. 3 we obtain $E_{F}-E_{0}=-0.50 \mathrm{eV}$. This is lower than the surface band minimum for $\mathrm{Au}(111) E_{F}-E_{0, \text { flat }}=$ $-0.46 \mathrm{eV}$, that we have measured separately on a different

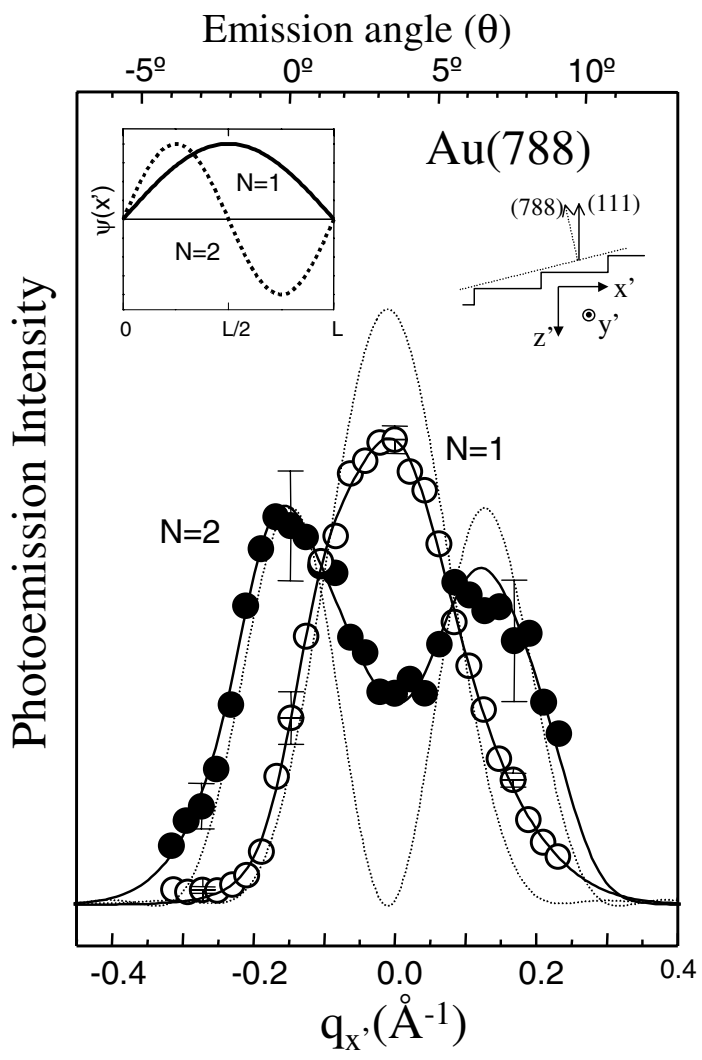

FIG. 4. Intensity of the two quantum levels $(N=1$, circles, $N=2$, dots) as a function of the emission angle perpendicular to the steps. $q_{x^{\prime}}$ represents the photoemission final state wave vector parallel to the (111) terrace, as indicated in the right inset. The solid lines are smooth lines that fit the data points. The dotted lines represent the expected photoemission intensity from $N=1$ and $N=2$ states of the infinite quantum well of width $L$, whose wave functions are shown in the left inset.

sample [12]. Note that $E_{0}$ in Eq. (1) corresponds to the surface state energy of an infinitely wide terrace such as those shown in Fig. 1, which has a reconstruction that is different from the normal $\mathrm{Au}(111)$ surface. Thus $E_{0}$ is given by the surface potential of a (111) plane with straight fcc- and hcp-packed domains of infinite length. For $\mathrm{Au}(111)$ the periodicity of the fcc-hcp superstructure is lower $(\sim 63 \AA)$ than that of the terraces in Fig. 1, and the domains are not straight but form a zigzag structure [13]. This represents a rougher surface for which we expect a higher value of $E_{0}$.

The intensity curves in Fig. 4 represent the experimental angle-resolved photoemission matrix element in the direction perpendicular to the steps for the terrace quantum levels. We can estimate such matrix element starting with Fermi's golden rule:

$$
I \propto\left|\left\langle\psi_{i}|\mathbf{A} \cdot \mathbf{p}| \psi_{f}\right\rangle\right|^{2} .
$$

At a terrace we can approach the initial state wave function as the product of a decaying wave into the bulk, a plane wave parallel to the terrace, and the wave function in the $x^{\prime}$ direction, i.e., $\psi_{i}\left(x^{\prime}, y^{\prime}, z^{\prime}\right)=e^{-\kappa z^{\prime}} \times e^{i k_{y^{\prime}} \cdot y^{\prime}} \times \psi_{k_{x^{\prime}}}\left(x^{\prime}\right)$. The $\left(x^{\prime}, y^{\prime}, z^{\prime}\right)$ reference system is depicted in Fig. 4 . The 
final state wave function can be assumed to be a plane wave in the $x^{\prime}$ and $y^{\prime}$ direction, and also in the $z^{\prime}$ direction when the electron is out of the crystal, thus $\psi_{f}=e^{i \mathbf{q} \cdot \mathbf{r}}$. With the photon energy used in the experiment and close to the surface normal $q_{z^{\prime}}\left(\gg q_{x^{\prime}}, q_{y^{\prime}}\right)$ is fixed by energy conservation. In the direction perpendicular to the steps $\left(q_{y^{\prime}}=0\right)$ we obtain $I\left(q_{x^{\prime}}\right)$, thus the angle-resolved photoemission matrix element for a given value of $q_{x^{\prime}}$ :

$$
I\left(q_{x^{\prime}}\right) \propto\left[\int e^{i q_{x^{\prime}} \cdot x^{\prime}} \psi_{k_{x^{\prime}}}\left(x^{\prime}\right) d x^{\prime}\right]^{2},
$$

where $q_{x^{\prime}}$ is the final-state wave vector along the $x^{\prime}$ direction, fixed by the emission angle. Note that $I\left(q_{x^{\prime}}\right)$ in Eq. (3) actually describes the probability density $\left|\psi\left(q_{x^{\prime}}\right)\right|^{2}$ in reciprocal space, which is the analog of the charge density mapped in real space by STM [4,5]. Such probability density is obtained experimentally in Fig. 4 for $N=1$ and $N=2$ in the terrace quantum well. It is thus interesting to compare the experimental probability density of the terrace quantum well with the infinite, one-dimensional quantum well of size $L$. In this case we have the known wave functions $\psi_{N}\left(x^{\prime}\right)=\sin \left(\pi N x^{\prime} / L\right)$ that allow to simplify Eq. (3) to give

$$
I_{Q W_{\infty}}\left(q_{x^{\prime}}\right)=A \times \frac{1-(-1)^{N} \cos \left(q_{x^{\prime}} L\right)}{\left[q_{x^{\prime}}^{2}-\left(\frac{\pi N}{L}\right)^{2}\right]^{2}} \times N^{2},
$$

where $A$ is a normalization constant which can be chosen to make the total intensity $\int I_{Q W_{\infty}}\left(q_{x^{\prime}}\right) d x^{\prime}$ equal to the area under the experimental intensity curve for $N=1$. With this value of $A$ we obtain the dotted lines shown in Fig. 4 for $N=1$ and $N=2$ of the infinite quantum well. Peak positions and widths are very well reproduced, indicating that terraces behave as infinite potential wells with similar wave functions. The deviations between $I_{Q W_{\infty}}\left(q_{x^{\prime}}\right)$ and the experimental $I\left(q_{x^{\prime}}\right)$ might be due to differences from the actual terrace potential [5]. Both terrace potential and wave functions could be obtained in real space from the experimental curve $I\left(q_{x^{\prime}}\right)$, as indicated by Eq. (3). However, $\sqrt{I\left(q_{x^{\prime}}\right)}$ gives only the modulus of the terrace quantum well wave function in momentum space. Its phase is needed to obtain the (experimental) wave function in real space by Fourier transformation. As we show elsewhere, this phase can be deduced self-consistently starting with the ansatz provided by the infinite potential well [14]. On the other hand, Eq. (3) disregards diffraction effects of the photoemission final state from the step array, that are expected to modulate $I\left(q_{x^{\prime}}\right)$. Diffraction from the superlattice is probably the reason for the asymmetry around $q_{x^{\prime}}=0$ observed in the data for both $N=1$ and $N=2$ in Fig. 4, since this asymmetry is found to be photon-energy dependent. Thus the intensity curves in Fig. 4 contain the essential information about the surface wave function at the $\mathrm{Au}(788)$ terrace quantum wells, that can be extracted with a refined theoretical approach of the angle-dependent photoemission matrix element including diffraction effects in the final state [14].
A. Mu., F.J. G. de A., and J.E. O. are supported by the Universidad del País Vasco (1/UPV/EHU/00057.240-EA8078/2000) and the Max Planck Research Award Program. V.R. and S.R. are supported by the CNRS-ULTIMATECH program, the CRIF, and the Université de Paris 7. A. Ma. is supported by a Marie Curie Fellowship of the European Union, under Contract No. HPMF-CT-200000565. V.P.-D. is supported by the Comunidad Autónoma de Madrid (Project No. 07N/0042/98) and the DGICYT (Spain) (Grant No. PB-97-1199). The experiments performed at LURE were funded by the Large Scale Facilities program of the European Union. Critical reading of the manuscript by F. J. Himpsel is acknowledged. Technical support from the Spanish-French beam line staff is gratefully acknowledged.

[1] F. J. Himpsel, J. E. Ortega, G. J. Mankey, and R. F. Willis, Adv. Phys. 47, 511 (1998).

[2] R. Nötzel and K. H. Ploog, Adv. Mater. 5, 22 (1993); R. Nötzel, Z. Niu, M. Ramsteimer, H. P. Schönherr, A. Trampert, L. Däweritz, and K. H. Ploog, Nature (London) 392, 56 (1998); P. Segovia, D. Purdie, M. Hegsberger, and Y. Baer, Nature (London) 402, 504 (1999).

[3] J.E. Ortega, S. Speller, A. Bachmann, A. Mascaraque, E. G. Michel, A. Mugarza, A. Närmann, A. Rubio, and F. J. Himpsel, Phys. Rev. Lett. 84, 6110 (2000).

[4] Ph. Avouris and I.-W. Lyo, Science 264, 942 (1994).

[5] L. Bürgi, O. Jeandupeux, A. Hirstein, H. Brune, and K. Kern, Phys. Rev. Lett. 81, 5370 (1998).

[6] X. Y. Wang, X. J. Shen, and R. M. Osgood, Jr., Phys. Rev. B 56, 7665 (1997).

[7] F. Baumberger, T. Greber, and J. Osterwalder, Phys. Rev. B 62, 15431 (2000).

[8] J. Viernow, J.-L. Lin, D. Y. Petrovykh, F. M. Leibsle, F. K- Men, and F. J. Himpsel, Appl. Phys. Lett. 72, 948 (1998).

[9] V. Repain, J. M. Berroir, B. Croset, S. Rousset, Y. Garreau, V. H. Etgens, and L. Lecoeur, Phys. Rev. Lett. 84, 5367 (2000).

[10] This is the average of the different values found in the literature, that vary from $0.24 \times m_{e}$ to $0.28 \times m_{e}$. The most recent photoemission study, S. LaShell, B. A. McDougall, and E. Jensen, Phys. Rev. Lett. 77, 3419 (1996), gives $0.25 \times m_{e}$.

[11] The data points in Fig. 3 display some random variation within the error bars, which can be interpreted as a narrow bandwidth. From this bandwidth we can estimate an upper limit for the transmission probability across the step barrier using a simple one-dimensional Kronig-Penney model. We obtain a maximum value of $|T|=0.1$ and $|T|=0.19$ for the first and the second levels, respectively.

[12] Our own measurement of $E_{0, \text { flat }}$ for $\mathrm{Au}(111)$ was also done at $300 \mathrm{~K}$ and agrees with the literature. See, for instance, R. Paniago, R. Matzdorf, G. Meister, and A. Goldmann, Surf. Sci. 336, 113 (1995).

[13] W. Chen, V. Madhavan, T. Jamneala, and M. F. Crommie, Phys. Rev. Lett. 80, 1469 (1998).

[14] F. J. García de Abajo et al. (to be published). 